University of Nebraska - Lincoln

DigitalCommons@University of Nebraska - Lincoln

\title{
Design considerations for table-top, laser-based VUV and X-ray free electron lasers
}

\author{
F. Gruner \\ Max-Planck-Institut für Quantenoptik, florian.gruener@physik.unl-muenchen.de \\ Stefan Becker \\ Universität Munchen, stefan.becker@physik.uni.muenchen.de \\ U. Schramm \\ Forschungszentrum Dresden-Rossendorf \\ T. Eichner \\ LMU München \\ R. Weingartner \\ Max-Planck-Institut für Quantenoptik \\ See next page for additional authors
}

Follow this and additional works at: https://digitalcommons.unl.edu/physicsfuchs

Gruner, F.; Becker, Stefan; Schramm, U.; Eichner, T.; Weingartner, R.; Habs, D.; Meyer-ter-Vehn, J.; Geissler, M.; Ferrario, M.; van der Geer, B.; Backe, H.; Lauth, W.; and Reiche, S., "Design considerations for table-top, laser-based VUV and X-ray free electron lasers" (2007). Matthias Fuchs Publications. 12.

https://digitalcommons.unl.edu/physicsfuchs/12

This Article is brought to you for free and open access by the Research Papers in Physics and Astronomy at DigitalCommons@University of Nebraska - Lincoln. It has been accepted for inclusion in Matthias Fuchs Publications by an authorized administrator of DigitalCommons@University of Nebraska - Lincoln. 


\section{Authors}

F. Gruner, Stefan Becker, U. Schramm, T. Eichner, R. Weingartner, D. Habs, J. Meyer-ter-Vehn, M. Geissler, M. Ferrario, B. van der Geer, H. Backe, W. Lauth, and S. Reiche 


\title{
Design considerations for table-top, laser-based VUV and X-ray free electron lasers
}

\author{
F. Grüner, ${ }^{1, *}$ S. Becker, ${ }^{1}$ U. Schramm, ${ }^{1}$ T. Eichner, ${ }^{1}$ R. Weingartner,${ }^{1}$ D. Habs,${ }^{1}$ J. Meyer-ter-Vehn, ${ }^{2}$ \\ M. Geissler, ${ }^{2}$ M. Ferrario,${ }^{3}$ L. Serafini,${ }^{3}$ B. van der Geer, ${ }^{4}$ H. Backe, ${ }^{5}$ W. Lauth,${ }^{5}$ and S. Reiche ${ }^{6}$ \\ ${ }^{1}$ Department of Physics, Ludwig-Maximilians-Universität München, 85748 Garching, Germany \\ ${ }^{2}$ Max-Planck Institute of Quantum Optics, 85748 Garching, Germany \\ ${ }^{3}$ INFN, 00044 Frascati, Italy \\ ${ }^{4}$ Pulsar Physics, 3762 Soest, The Netherlands \\ ${ }^{5}$ Institute of Nuclear Physics, Mainz, Germany \\ ${ }^{6}$ University of California Los Angelos, UCLA, Los Angelos, USA
}

(Dated: 13.12.2006)

\begin{abstract}
A recent breakthrough in laser-plasma accelerators, based upon ultrashort high-intensity lasers, demonstrated the generation of quasi-monoenergetic GeV-electrons. With future Petawatt lasers ultra-high beam currents of $\sim 100 \mathrm{kA}$ in $\sim 10$ fs can be expected, allowing for drastic reduction in the undulator length of free-electron-lasers (FELs). We present a discussion of the key aspects of a table-top FEL design, including energy loss and chirps induced by space-charge and wakefields. These effects become important for an optimized table-top FEL operation. A first proof-of-principle VUV case is considered as well as a table-top X-ray-FEL which may open a brilliant light source also for new ways in clinical diagnostics.
\end{abstract}

PACS numbers: 41.60.Cr,52.38.Kd

\section{INTRODUCTION}

The pursuit of table-top FELs combines two rapidly developing fields: laser-plasma accelerators, where the generation of intense quasi-monoenergetic electron bunches up to the $\mathrm{GeV}$ range has been achieved [1, 2, 3, 4], and on the other hand large-scale X-ray free-electron lasers (XFELs) that are expected to deliver photon beams with unprecedented peak brilliance. A prominent application of such FEL pulses is single molecule imaging [5]. The proposed laser-plasma accelerator-based FELs would not only allow a greater availability due to their smaller size and costs, but also offer new features, such as pulses synchronized to the phase-controlled few-cycle driver laser 6] for pump-probe experiments. Moreover, the X-ray energy of a table-top XFEL can be as large as required for medical diagnostics (above $20 \mathrm{keV}$ [7]).

The mechanism for the generation of intense $(\mathrm{nC}$ charge) quasi-monoenergetic electron pulses by laserplasma accelerators requires an ultrashort, high-intensity laser-pulse with a length shorter than the plasma wavelength (on the $\mu \mathrm{m}$-scale corresponding to gas densities of $\left.10^{19} \mathrm{~cm}^{-3}\right)$. Due to the ponderomotive force, plasma electrons are blown out transversely, leaving an electronfree zone - the bubble - behind the laser pulse [8]. These electrons return to the axis after half a plasma oscillation, thus determining the size of the bubble in the order of the plasma wavelength. Typically about $10^{9} \ldots 10^{10}$ electrons are captured into the bubble, as found both experimentally and from scaling laws of relevant bubble parameters within the framework of similarity arguments [9]. Due to the inertial positive ion background, these electrons experience a strong electrical field gradient of up to TV/m. Particle-in-cell (PIC) simulations [10] show that the bubble electrons form a stem that is geometrically considerably smaller than the bubble as seen in Fig. 1.

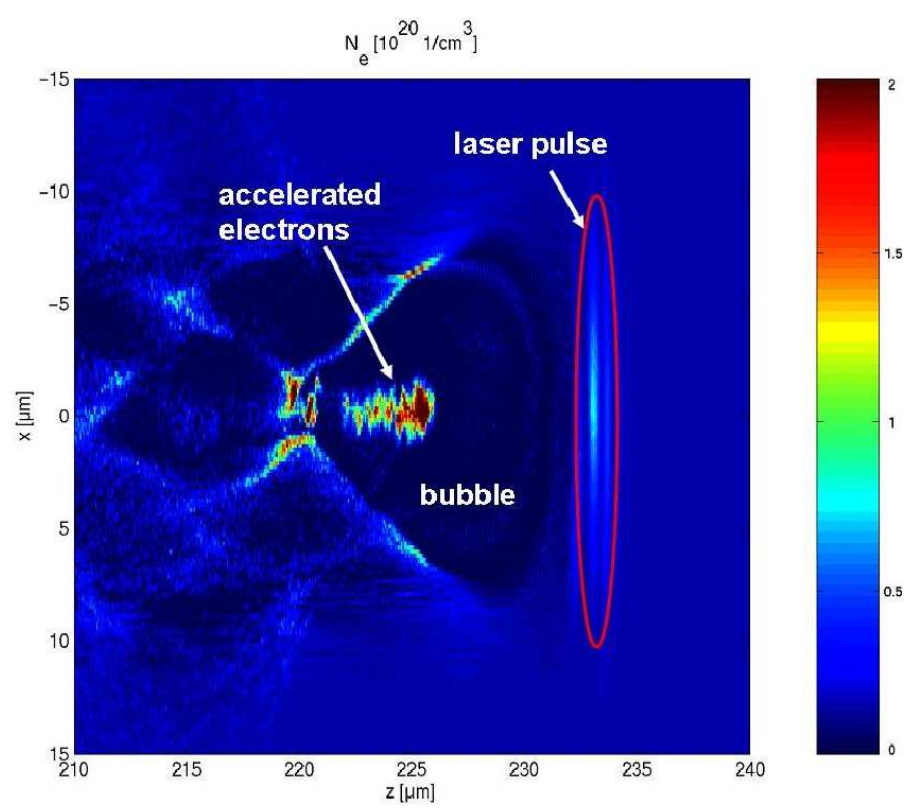

FIG. 1: (Color online) Snapshot of electron density (in units of $10^{20} \mathrm{~cm}^{-3}$ ) from PIC simulation. The geometrical size of the electron-free cavity ("bubble") behind the laser spot corresponds to the plasma period, which is about $8 \mu \mathrm{m}$ in this case (gas density $1.8 \cdot 10^{19} \mathrm{~cm}^{-3}$ ). The stem of the highenergy electrons is much shorter than the plasma period.

For these dimensions, beam currents of the order of $\sim 100 \mathrm{kA}$ (i.e. $\sim 1 \mathrm{nC}$ charge within $\sim 10 \mathrm{fs}$ ) can be reached without the need for any bunch compressor.

For preparation of the required gas densities, either supersonic gas-jets or capillaries [11] are used, the latter consisting of $\mathrm{a} \sim 300 \mu \mathrm{m}$-thin gas-filled channel with a 
parabolic radial ion density profile generated by a second laser pulse or discharge. The advantage of the capillary is that the laser can be guided beyond the Rayleigh length allowing longer acceleration distances and hence higher electron energies. The very recent experiments by Leemans et al. [4], utilizing a 40-TW laser pulse of $38 \mathrm{fs}$ duration and a gas density of $4.3 \cdot 10^{18} \mathrm{~cm}^{-3}$, clearly show that $1 \mathrm{GeV}$ electron beams can now be produced with capillaries. However, the measured charge of $30 \mathrm{pC}$ is significantly below our design goal of $1 \mathrm{nC}$. In order to further improve the resulting current, we propose the scheme described below. The number of bubble electrons can be increased when using plasmas with higher density such as in Fig. 1, because the feeding process is more efficient if more plasma electrons are present. An increased gas density requires shorter laser-pulses (sub-10-fs), such that the entire laser pulse fits into one plasma period. In this case, the entire laser pulse energy can be used, while longer pulses lose energy during self-shortening for entering the bubble regime. Therefore, in contrast to all previous laser-acceleration experiments, we propose to use a different driver laser, namely a $\sim 5$ fs-Petawatt laser (like the Petawatt-Field-Synthesizer PFS at MPQ [12]). Own PIC simulations show that such ultrashort lasers can capture more than $1 \mathrm{nC}$ charge inside the bubble. The smaller plasma period leads to smaller bubbles, hence shorter bubble stems and thus higher currents (1 $\mathrm{nC}$ in $10 \mathrm{fs}$ ). However, the expected final energy in the bubble regime is, according to the scaling laws, lower for shorter laser pulses. To overcome this limit, we also propose to use a capillary and suggest the following scenario therein: with a longitudinal plasma density gradient, the laser pulse can be forced to gradually increase its diameter, thus adiabatically turning from the pure bubble regime into the pure blow-out regime long before the laser is depleted. Fig. 1 shows a snapshot of a bubble around the transition from the bubble to the blow-out regime, where the electrons blown away from the laser and flowing around the bubble are so strongly deflected by the electric field of the captured bubble electons, that electrons are no more scattered into the bubble (which is not charge-neutralized yet). The number and absolute energy spread of the bubble electrons is thus frozen, but their energy is still increased due to the present bubble fields. The remaining energy of the laser allows to maintain the bubble structure for the remaining distance inside the capillary, where the laser is then guided along to overcome the Rayleigh limit. Only for this stage with increased laser beam size, the capillary-induced guiding is relevant. Note that this scheme is a two-stage approach, but within one and the same capillary, where the laser turns adiabatically from one stage into the other. It can be expected that the energy spread for $100 \mathrm{MeV}$ is about $1 \%$ (as confirmed by measurements [2]), but $0.1 \%$ for 1 $\mathrm{GeV}$ [13]. Normalized emittances are found both from simulations and experiments to range between $0.1-1$ $\mathrm{mm} \cdot \mathrm{mrad}$.

\section{TABLE-TOP FREE-ELECTRON-LASERS}

An FEL requires an undulator which is an arrangement of magnets with an alternating transverse magnetic field. Electrons in an undulator are forced on a sinusoidal trajectory and can thus couple with a co-propagating radiation field. The induced energy modulation yields a current modulation from the dispersion of the undulator field. This modulation is called micro-bunching expressing the fact that the electrons are grouped into small bunches separated by a fixed distance. Therefore, electrons emit coherent radiation with a wavelength equal to the periodic length between the micro-bunches. In a Self-Amplification of Spontaneous Emission (SASE) FEL, there is no initial radiation field and the seed has to be built up by the spontaneous (incoherent) emission 14.

We present quantitative arguments, which are complemented by SASE FEL (GENESIS 1.3 [15]) simulations. The gain length, which is the e-folding length of the exponential amplification of the radiation power, is

$$
L_{\text {gain }, \text { ideal }}=\frac{\lambda_{u}}{4 \pi \sqrt{3} \rho},
$$

with undulator period $\lambda_{u}$ and the basic scaling parameter $\rho$. This Pierce or FEL parameter describes the conversion efficiency from the electron beam power into the FEL radiation power and reads for the one-dimensional and ideal case (neglecting energy spread, emittance, diffraction, and time-dependence) [14, 16]

$$
\rho=\frac{1}{2 \gamma}\left[\frac{I}{I_{A}}\left(\frac{\lambda_{u} A_{u}}{2 \pi \sigma_{x}}\right)^{2}\right]^{1 / 3} .
$$

Here $\gamma=E_{\text {beam }} / m c^{2}$ is the electron beam energy, $I$ the beam current, $I_{A}=17 k A$ the Alfven-current, $\sigma_{x}$ the beam size and $A_{u}=a_{u}\left[J_{0}(\zeta)-J_{1}(\zeta)\right]$ (planar undulator), whereby $a_{u}^{2}=K^{2} / 2, K$ the undulator parameter $\left(K=0.93 \cdot \lambda_{u}[\mathrm{~cm}] \cdot B_{0}[T]\right.$ and $B_{0}$ the magnetic field strength on the undulator axis), $\zeta=a_{u}^{2} /\left(2\left(1+a_{u}^{2}\right)\right)$, and $J$ are Bessel functions. In presence of energy spread, emittance, and diffraction, a correction factor $\Lambda$ is introduced for the gain length [16]: $L_{\text {gain }}=L_{\text {gain,ideal }}(1+\Lambda)$. The saturation length $L_{\text {sat }}$ is the length along the undulator at which maximum micro-bunching is reached. The power of the FEL radiation at this point is the saturation power $P_{s a t}$, while the shot noise power $P_{n}$ is the power of the spontaneous undulator radiation from which the SASE FEL starts. The coupling factor $\alpha=1 / 9$ describes the efficiency at which the noise power couples to the FEL gain process. The saturation length reads 


$$
L_{\text {sat }}=L_{\text {gain }} \cdot \ln \left(\frac{P_{\text {sat }}}{\alpha \cdot P_{n}}\right),
$$

where the saturation power scales as

$$
P_{\text {sat }} \sim\left(\frac{1}{1+\Lambda}\right)^{2}\left(I \cdot \lambda_{u}\right)^{4 / 3}
$$

The FEL-wavelength is, in case of a planar undulator,

$$
\lambda=\frac{\lambda_{u}}{2 \gamma^{2}}\left[1+\frac{K^{2}}{2}\right] .
$$

Thus, for reaching a certain wavelength $\lambda$, a shorter undulator period $\lambda_{u}$ allows using less energetic electrons. In the following Tab. 1 and Fig. 2, we compare the FLASH VUV FEL (DESY) in the so-called femtosecond mode [17] with our corresponding table-top VUV FEL as well as a table-top XFEL operating with $1.2 \mathrm{GeV}$ electrons, discussed further below.

TABLE I: Parameters for the comparison between the DESY femtosecond-mode Flash-VUV case and the corresponding table-top VUV FEL as well as a table-top X-ray FEL (rms values).

\begin{tabular}{|l|c|c|c|}
\hline Parameter & FLASH (fs) & TT-VUV-FEL & TT-XFEL \\
\hline current & $1.3 \mathrm{kA}$ & $50 \mathrm{kA}$ & $160 \mathrm{kA}$ \\
norm. emitt. & $6 \mathrm{~mm} \cdot \mathrm{mrad}$ & $1 \mathrm{~mm} \cdot \mathrm{mrad}$ & $1 \mathrm{~mm} \cdot \mathrm{mrad}$ \\
beam size & $170 \mu \mathrm{m}$ & $30 \mu \mathrm{m}$ & $30 \mu \mathrm{m}$ \\
energy & $461.5 \mathrm{MeV}$ & $150 \mathrm{MeV}$ & $1.74 \mathrm{GeV}$ \\
energy spread & $0.04 \%$ & $0.5 \%$ & $0.1 \%$ \\
und. period & $27.3 \mathrm{~mm}$ & $5 \mathrm{~mm}$ & $5 \mathrm{~mm}$ \\
wavelength & $30 \mathrm{~nm}$ & $32 \mathrm{~nm}$ & $0.25 \mathrm{~nm}$ \\
Pierce par. & 0.002 & 0.01 & 0.0015 \\
sat. length & $19 \mathrm{~m}$ & $0.8 \mathrm{~m}$ & $5 \mathrm{~m}$ \\
pulse length & $55 \mathrm{fs}$ & $4 \mathrm{fs}$ & $4 \mathrm{fs}$ \\
sat. power & $0.8 \mathrm{GW}$ & $2.0 \mathrm{GW}$ & $58 \mathrm{GW}$ \\
\hline
\end{tabular}

The importance of the ultra-high current in the tabletop case is evident. The smaller undulator period allows a smaller beam energy, hence decreasing the gain and saturation lengths. The Pierce parameter gives the upper limit of the acceptable energy spread $\sigma_{\gamma} / \gamma$. Thus, for compensating the relatively large energy spread of laserplasma accelerators and for maintaining a large output power, a beam current significantly above $\sim 17 \mathrm{kA}$ is mandatory for keeping $\rho$ large and $\Lambda$ small.

\section{Space charge effects}

Such ultra-high beam currents are subject to strong space-charge forces. After release into vacuum, the electron bunch starts expanding, for which there are two

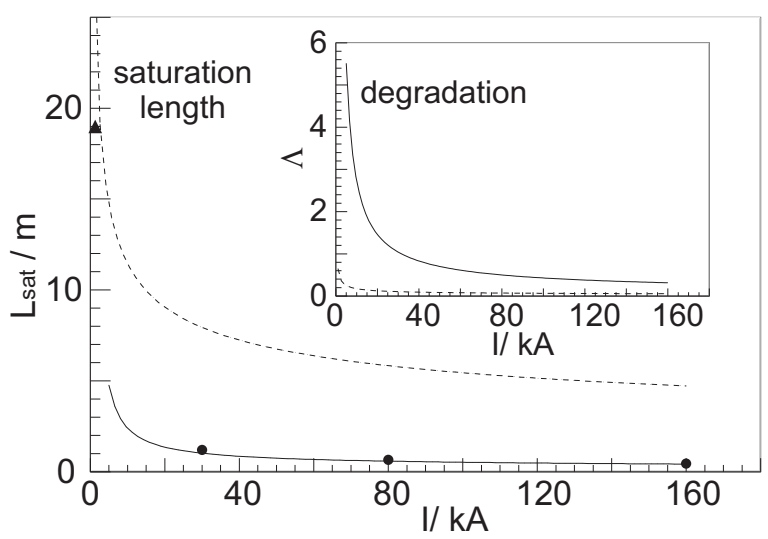

FIG. 2: Saturation lengths (Eq. 3) and (inset) degradation factor $\Lambda$ as a function of electron beam current $I$ : DESY's FLASH (dashed curve, triangle) and the table-top VUV scenario (solid line). The circles denote specific GENESIS runs.

sources: (i) its own space-charge, driving a Coulombexplosion, i.e. (transverse) space-charge expansion and (longitudinal) debunching, and (ii) its initial divergence, which drives a linear transverse expansion. Such expansions transform potential energy into kinetic, hence changing the initial energy distribution and bunch form. First, we want to discuss an extreme case, that is, an upper limit of the charge that can be expected from a laser-plasma accelerator at lower energies. For this study we take a beam energy of $\gamma_{0}=260,1.25 \mathrm{nC}$ charge, and a Gaussian bunch with sizes $\sigma_{x, y, z}=1 \mu \mathrm{m}$, corresponding to $I=150 \mathrm{kA}$, and an initial divergence of $\theta_{0}=1$ mrad. In the rest frame of the bunch, its length amounts to $\sigma_{z}^{\prime}=\sigma_{z} \cdot \gamma_{0}=260 \mu \mathrm{m}$. For such an aspect-ratio, the transverse Coulomb-explosion dominates over the longitudinal one (GPT [18] simulations reveal that after a time period of 1 ps $\sigma_{x}^{\prime}=73 \mu \mathrm{m}$, while $\left.\sigma_{z}^{\prime}=268 \mu \mathrm{m}\right)$. Fig. 3 shows the electron distribution in the bunch rest frame from the GPT simulations.
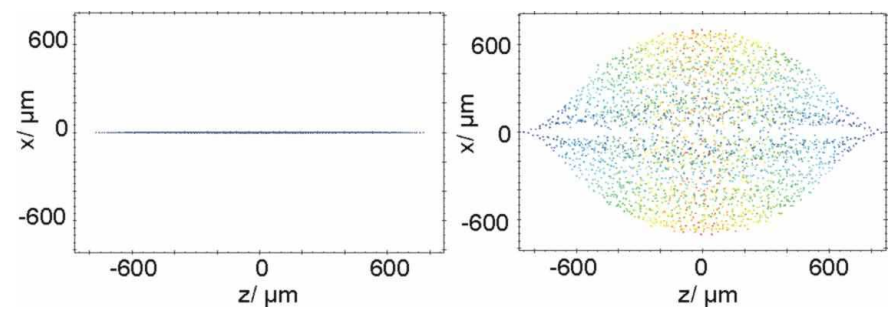

FIG. 3: (Color online) Projected spatial electron distribution within the bunch rest frame in the extreme case, at $\tau=0 \mathrm{~s}$ (left) and $\tau=4 \mathrm{ps}$ (right), from a GPT run. The color code indicates the electron energy (blue is initial kinetic energy, red is increased energy). The transverse expansion is clearly dominating.

It can be seen that the transverse Coulomb explosion dominates the longitudinal one. We want to em- 
phasize here that studies of space-charge effects of such ultra-dense relativistic bunches can be simulated most accurately only with codes (such as GPT and CSRtrack [19]) which utilize point-to-point interactions (a result also found in Ref. [20]). In contrast, other codes merely based upon Poisson-solvers cannot cope with large relative particle motions within the bunch rest frame in case of large initial energy spreads and Coulomb explosioninduced motion.

In comparison with the Coulomb-driven explosion, the linear divergence-driven transverse expansion is even larger: after a distance of $1 \mathrm{~cm}$ in the laboratory frame, the beam size is increased to $10 \mu \mathrm{m}$, while transverse Coulomb-explosion yields $6 \mu \mathrm{m}$ only. Hence, even in the extreme case the transverse bunch expansion is dominated by the initial divergence and for lower charges this dominance increases. If $\gamma_{0}$ is the bunch energy just before release into vacuum, $\gamma^{\prime}$ and $\beta^{\prime}$ the energy and velocity of a test electron in the bunch rest frame, $\gamma$ and $\beta \approx 1$ its laboratory frame energy and velocity, then one can clearly distinguish between a transverse-dominated expansion and a longitudinal-dominated one: in the first case, where the electron moves in the rest frame with velocity $\pm \beta^{\prime}$ along transverse direction, $\gamma=\gamma_{0} \cdot \gamma^{\prime}$, while in the latter case, where the electron moves with velocity $\pm \beta^{\prime}$ in beam direction, $\gamma=\gamma_{0} \cdot \gamma^{\prime} \cdot\left(1 \pm \beta^{\prime}\right)$. Hence, in a purely transverse expansion all electrons gain kinetic energy, while in a purely longitudinal expansion, some electrons lose energy. The inset of Fig. 4 shows the absolute kinetic electron energy distribution along the bunch at a distance of $z=3 \mathrm{~cm}$ after its release into vacuum (in case of $0.6 \mathrm{nC}$ ). This spectrum shows that the bunch under study undergoes mostly a transverse expansion. The maximum energy gain lies in the middle of the bunch, where the density is highest, i.e., where initially the highest potential energy was stored. That the space-charge driven transverse expansion is weaker than the divergence-driven one is also confirmed by the fact that (even in the extreme case) the divergence is increased only to $1.3 \mathrm{mrad}$. After passing a focusing system (a triplet of quadrupoles, positioned at $z=4 \mathrm{~cm}$, having a length of about $10 \mathrm{~cm}$ ), the Gaussian-like energy distribution is transformed into a quasi-linear one, as depicted in Fig. 4. The reason is that in the resulting quasi-collimated low-divergence beam faster electrons can catch up the slower ones. Note that after $z=3 \mathrm{~cm}$ (inset) the fastest electrons are symmetrically distributed around the middle of the bunch. These electrons start overtaking the slower ones, that is, they move forward within the bunch. If initially longitudinal debunching dominates, the fastest electrons are found in the head and the slowest ones in the tail of the bunch.

A key parameter for SASE to occur is the energy spread $\sigma_{\gamma} / \gamma$, which must always be smaller than the Pierce parameter $\rho$. The relevant energy spread is taken over a bunch slice with the size of one cooperation

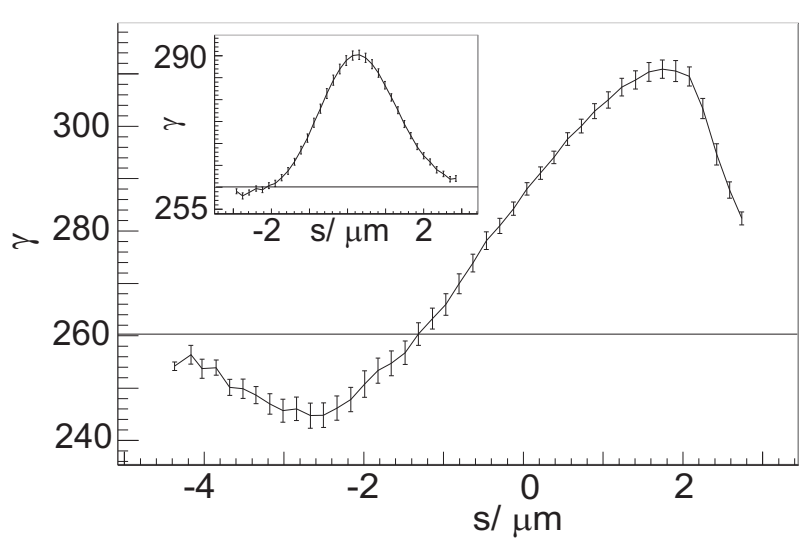

FIG. 4: Energy vs. position (in co-moving frame) along the bunch before entering the undulator (GPT simulation): the Gaussian-like distribution after a drift of $z=3 \mathrm{~cm}$ behind the gas-jet (inset), indicating a transverse-dominated bunch expansion, is stretched into a quasi-linear one behind the focusing system. The vertical lines show the slice energy spread $\sigma_{\gamma}$ and the horizontal lines mark the initial energy $\gamma_{0}=260$.

length, i.e. the slippage length of the radiation along the bunch over one gain length. As seen in Fig. 4, a large fraction of the bunch fulfills $\sigma_{\gamma} / \gamma<\rho$. Due to the slippage of the radiation relative to the bunch, the linear energy chirp must be compensated, because the energy factor $\gamma$ in Eq. (5) increases along the bunch. However, in case of short-period undulators the gap is correspondingly small (typically one third of the undulator period) and such small gaps lead to strong wakefields also causing an energy variation along the bunch [24]. In case of ultrashort bunches, the characteristic length of the resistive wake potential is longer than the bunch length and, hence, the energy change is found to be negative with a linear few-percent variation along the bunch. This in turn implies that the bunch as a whole is decelerated during the passage of the undulator, whereby the head loses less energy than the tail. It turns out that the linear energy chirp induced by space-charge corresponds well with the wakefield-induced slowing down of the entire electron bunch. Therefore, the radiation slipping in forward direction interacts with electrons of effectively constant energy, if by varying the gap the bunch energy loss is tuned with respect to the slippage. In other words, the space-charge effects and the wakefield effects cancel each other. We have found with simulations that for a first proof-of-principle of a table-top FEL the comparably low energy of $130-150 \mathrm{MeV}$ is well suited to cope with the wakefields in the undulator. In this sense the table-top VUV case given in Tab. 1 can be regarded as an optimal test case.

Coherent synchrotron radiation (CSR) 21] as another source for increasing the (slice) energy spread within the undulator was found to have negligible impact. This 
was shown with CSRtrack simulations which take into account that in our cases the bunches have a much larger transverse size $\left(\sigma_{x, y} \sim 30 \mu \mathrm{m}\right)$ than length $\left(\sigma_{z} \sim 1 \mu \mathrm{m}\right)$.

\section{Table-top XFEL}

So far we have discussed a proof-of-principle scenario at relatively low electron energies, where space-charge effects play a dominant role leading to a linear energy chirp. The situation of the proposed table-top X-FEL (TT-XFEL) is different. For reaching a wavelength of $\lambda=0.25 \mathrm{~nm}$, an electron energy of $1.74 \mathrm{GeV}$ is needed in case of a period of $\lambda_{u}=5 \mathrm{~mm}$. Space charge effects are much weaker here. For this less demanding situation we have confirmed with four different simulation codes (GPT, ASTRA [20], CSRtrack, HOMDYN [23]) that above $1 \mathrm{GeV}$, with the same parameters as in the extreme case given above, Coulomb-explosion leads to a projected energy chirp of below $0.3 \%$ and a bunch elongation with a factor below 1.1. Furthermore, CSR can be neglected in agreement with the 1D theory. Experimentally the most demanding constraint is that the Pierce parameter is one order of magnitude smaller than in the test case at $130 \mathrm{MeV}$, hence the electron (slice) energy spread should be as small as $0.1 \%$. This goal seems to be within reach considering the bubble-transformation scenario mentioned above.

Without the effect of wakefields GENESIS simulations have shown that this TT-XFEL scenario with an undulator length of only $5 \mathrm{~m}$ yields $8 \cdot 10^{11}$ photons/bunch within $\sim 4$ fs and $0.2 \%$ bandwidth, a divergence of $10 \mu \mathrm{rad}$, and a beam size of $20 \mu \mathrm{m}$. However, the wakefields become the dominant degrading effect as the required undulator length is larger and the Pierce parameter reduced, hence also the tolerance with respect to variations in the electron energy. But since there is no initial space-chargeinduced energy chirp, one must find another method for compensating the wakefield-induced energy variation. A suitable method for compensating the wakefields for the TT-XFEL would be tapering, i.e., varying the undulator period along the undulator. Due to the fact that the undulator parameter $K$ is smaller than unity, tapering via $K$, i.e., by gap variation, could only be used as finetuning. Depending on the specific material properties of the undulator surface, our first wake calculations show that the bunch center loses over the entire $5 \mathrm{~m}$ undulator length about $10 \%$ of its initial energy. Due to the linear wake field variation along the bunch only a fraction of it will undergo SASE. A detailed study will be further investigated in a future paper.

\section{Experimental Status}

In order to demonstrate practical feasibility, we have built and tested a miniature focusing system and undulator consisting of permanent magnets. The focusing triplet consists of mini-quadrupoles with an aperture of just $5 \mathrm{~mm}$, hence allowing for measured gradients of 530 $\mathrm{T} / \mathrm{m}$. Their focusing strength was measured with a 600 $\mathrm{MeV}$ electron beam (at Mainz Microtron facility MAMI, Mainz, Germany). A first test hybrid undulator with a period of only $5 \mathrm{~mm}$ and a peak magnetic field strength of $\sim 1 \mathrm{~T}$ has been built and produced with a $855 \mathrm{MeV}$ beam (also at MAMI) an undulator radiation spectrum as expected. These results will be published elsewhere.

\section{CONCLUSION}

We have shown by means of analytical estimates and SASE FEL simulations that laser-plasma acceleratorbased FELs can be operated with only meter-scale undulators. The key parameter is the ultra-high electron peak current, which significantly reduces the gain length and increases the tolerance with respect to energy spread and emittance. The latter would also allow to increase the TT-XFEL photon energy into the medically relevant range of $20-50 \mathrm{keV}$, because the limiting quantum fluctuations in the spontaneous undulator radiation scale with $\gamma^{4}[25]$ and the required electron energy of the TT-XFEL is comparatively small.

We thank W. Decking, M. Dohlus, K. Flöttmann, T. Limberg, and J. Rossbach (all DESY) for fruitful discussions. Supported by Deutsche Forschungsgemeinschaft through the DFG-Cluster of Excellence Munich-Centre for Advanced Photonics. This work was also supported by DFG under Contract No. TR18.

* Electronic address: florian.gruener@physik.uni-muenchen.de

[1] S.P.D. Mangles et al., Nature 431, 535 (2004)

[2] C.G.R. Geddes et al., Nature 431, 538 (2004)

[3] J. Faure et al.,Nature 431, 541 (2004)

[4] W.P. Leemans et al., Nature Physics 2, 696 (2006)

[5] R. Neutze et al., Nature 406, 752 (2000)

[6] A. Baltuska et al., Nature 421, 611 (2003)

[7] W. Thomlinson, P. Suortti, and D. Chapman, Nucl. Instrum. Methods Phys. Res. A 543, 288 (2005)

[8] A. Pukhov and J. Meyer-ter-Vehn, Appl. Phys. B 74, 355 (2002)

[9] A. Pukhov and S. Gordienko, Phil. Trans. R. Soc. A 364, 623 (2006)

[10] M. Geissler, J. Schreiber, and J. Meyer-ter-Vehn, New J. Phys. 8186 (2006)

[11] D.J. Spence, A. Butler, and S. Hooker, J. Opt. Soc. Am. B 20, 138 (2003)

[12] http://www.attoworld.de/research/PFS.html 
[13] Note that the detector resolution in the cited GeVexperiment did not suffice to resolve energy spreads below one percent

[14] R. Bonifacio, C. Pellegrini, and L.M. Narduci, Opt. Comm. 50, 373 (1984)

[15] S. Reiche, Nucl. Instrum. Methods Phys. Res. A 429, 243 (1999)

[16] M. Xie, Nucl. Instrum. Methods Phys. Res. A 445, 59 (2000)

[17] Technical Design Report: http://www-hasylab.desy.de/facility/fel/vuv/

[18] M.J. de Loos, S.B. van der Geer, Proc. 5th Eur. Part. Acc. Conf., Sitges, 1241 (1996)

[19] M. Dohlus and T. Limberg, Proceedings of the FEL 2004 Conference, 18-21
[20] G. Fubiana, J. Qiang, E. Esarey, and W.P. Leemans, Phys. Rev. ST Accel. Beams 9, 064402 (2006)

[21] E. Saldin, E.A. Schneidmiller, and M.V. Yurkov, Nucl. Instrum. Methods Phys. Res. A 417, 158 (1998)

[22] K. Floettmann, Astra User's Manual, 2000, http://www.desy.de mpyflo/

[23] M. Ferrario, J. E. Clendenin, D. T. Palmer, J. B. Rosenzweig, L. Serafini, Proc. of the 2nd ICFA Adv. Acc. Workshop on "The Physics of High Brightness Beams", UCLA, Nov., 1999; see also SLAC-PUB-8400

[24] A.W. Chao, Physics of collective beam instabilities in high energy accelerators (John Wiley \& Sons, New York, 1993); K. Bane, SLAC-PUB-11829 (2006)

[25] M. Sands, Phys. Rev. 97, 470 (1955) 